Ebisu

Études japonaises

Études japonaises

47 | printemps-été 2012

Catastrophes du 11 mars 2011, désastre de

Fukushima : fractures et émergences

\title{
Cataclysme et pouvoir politique dans l'imaginaire au Japon : l'exemple des namazu-e du séisme de l'ère Ansei (1855)
}

Cataclysm and Politics Within the Japanese Imagination: Namazu-e of the Ansei-Edo Earthquake

日本において想像がつくり出した世界の中の大異变と政治一安政江 戸地震の鯰絵

\section{Julien Bernardi-Morel}

\section{OpenEdition}

\section{Journals}

Édition électronique

URL : http://journals.openedition.org/ebisu/516

DOI : 10.4000/ebisu.516

ISSN : 2189-1893

\section{Éditeur :}

Institut français de recherche sur le Japon (UMIFRE 19 MAEE-CNRS), Maison franco-japonaise

\section{Édition imprimée}

Date de publication : 1 juin 2012

Pagination : 255-266

ISSN : 1340-3656

Référence électronique

Julien Bernardi-Morel, « Cataclysme et pouvoir politique dans l'imaginaire au Japon : l'exemple des namazu-e du séisme de l'ère Ansei (1855) », Ebisu [En ligne], 47 | printemps-été 2012, mis en ligne le 21 mai 2014, consulté le 22 avril 2019. URL : http://journals.openedition.org/ebisu/516 ; DOI

10.4000/ebisu.516 


\section{Cataclysme et pouvoir politique dans l'imaginaire au Japon}

L'exemple des namazu-e du séisme de l'ère Ansei (1855)

Julien BERNARDI-MOREL

Le 11 novembre 1855, un séisme dévastateur frappa la ville d'Edo (ancien nom de Tokyo). L'épicentre du tremblement de terre près du cœur de la cité causa des dégâts incommensurables. Le nombre de victimes est estimé entre 7000 et 10000 (Usami 2003 : 171-182). Les dégâts matériels furent eux aussi très sévères, puisque le séisme et les incendies détruisirent plus de 14000 bâtiments. Le nombre important de blessés, les dégâts, les menaces persistantes d'incendies et surtout le fait que la capitale politique du Japon ait été touchée accentuèrent l'impact psychologique du séisme.

Consciente ou inconsciente, une question demeurait dans tous les esprits : qui blâmer?

Des théories d'interventions cosmiques délibérées au sein de sociétés étaient présentes dans de nombreuses philosophies comme le confucianisme ou la pensée tentō 天道 ${ }^{1}$. En effet, avant le Xx ${ }^{\mathrm{e}}$ siècle, en Asie du Sud-Est, les

Après une licence et un master en langue, littérature et civilisation du Japon, Julien Bernardi-Morel entame des recherches sur les cataclysmes et le motif du héros dans l'imaginaire du Japon. Il occupe depuis 2011 un poste de lecteur à l'université Kinjō gakuin 金城学院大学 de Nagoya.

1. Le tentō est un culte syncrétique basé sur des idées religieuses et philosophiques provenant de Chine. Inspiré du taoïsme et du confucianisme, le terme servit d'abord à désigner par métonymie les divinités indo-bouddhiques Bonten (Brahmā) et Taishakuten (Indra), mais aussi Amaterasu, divinité centrale de la croyance shintô. Plus tard, il évolua pour désigner l'ensemble des divinités célestes, une autorité divine supérieure au monde terrestre. 
théories concernant les tremblements de terre étaient pour la plupart liées à la notion des cinq agents du Yin et du Yang qui se manifestaient dans l'univers à travers cinq éléments : l'eau, le feu, le métal, l'air et le bois. Des penseurs chinois développèrent ainsi des théories très élaborées de correspondances cosmologiques au sein desquelles ces cinq éléments jouaient un rôle clé dans l'explication des phénomènes naturels. Plus tard, l'idée de base du Yin et du Yang opérant un équilibre régulé par les cinq éléments devint extrêmement répandue parmi les populations d'Asie.

À partir des années 1830, le Japon connut une série de désastres naturels, d'épidémies et de famines qui entraînèrent des soulèvements populaires $^{2}$. Ces catastrophes commentées par les médias de l'époque $e^{3}$ poussèrent progressivement la majorité des Japonais à voir dans ces désastres et en particulier dans les séismes la résultante d'un déséquilibre entre ces cinq éléments du Yin et du Yang à l'intérieur de la terre. Malgré certains détails mineurs qui pouvaient être l'objet de désaccords, ce concept faisait partie depuis longtemps d'un sens commun intellectuel et social, mais l'idée générale que des forces cosmiques interviennent dans une société en perdition afin de corriger les erreurs humaines ne devint claire pour la population japonaise que très tardivement. En effet, peu avant la catastrophe de 1855, le pays venait de faire l'expérience de nombreux tremblements de terre, tels que ceux de Shinano, d'Odawara, d'Iga-Ueno (actuel département de Mie), d'Ise et de Konoe (actuel département de Shiga), qui avaient détruit plus de 20000 habitations et fait des milliers de victimes (Noguchi 2004 : 34-37). L'année de la seconde venue au Japon de la flotte du commodore Perry, en 1854, deux séismes sous-marins firent trembler la côte Pacifique à deux jours d'intervalle. À la fin de l'année 1854, le tremblement de terre d'Ansei Tōkai 安政東海 frappa une région allant d'Edo à la baie d'Ise et le séisme d'Ansei Nankai 安政南海, la région d'Osaka (Noguchi 2004 : 39). Au lendemain de ces catastrophes, un des principaux dirigeants du

2. En effet, durant l'ère Tenpō (1830-1844), l'accumulation de ces catastrophes provoqua des émeutes, en particulier celle menée par un ancien fonctionnaire de police, Ōshio Heihachirō 大塩平八郎 (1793-1837), qui eut lieu à Osaka en 1837.

3. La presse écrite de l'époque ou kawaraban 瓦版 reposait sur un système complexe de signes, de symboles, d'analogies et de métaphores qui existaient dans un contexte quotidien et croisaient sans efforts les frontières de la science, de l'ésotérisme, des croyances populaires et des idéologies politiques. 
bakufu, le seigneur Matsudaira Shungaku 松平春猋 (1828-1890), cousin du shogun, réagit par une lettre envoyée à son supérieur Abe Masahiro 阿部正弘 (1819-1857) ${ }^{4}$, dans laquelle il lista les différentes catastrophes, désastres et récentes visites intempestives des vaisseaux américains, anglais et russes. Il en conclut alors que ces différents événements " constituaient définitivement un rappel à l'ordre divin " (Noguchi 2004 : 39).

Dans une société où le gouvernement consistait en une organisation militaire exerçant une hégémonie totale d'une part et un empereur à Kyoto possédant un rôle surtout religieux et culturel d'autre part, la divergence entre morale et pouvoir politique ne faisait que s'accroître, et les phénomènes atmosphériques, les mauvaises récoltes, les épidémies devinrent alors le ciment des manifestations d'un "mécontentement " divin. Le séisme d'Edo pendant l'ère Ansei 安政 sembla faire trembler en même temps que la croûte terrestre, les fondations même de la société d'Edo.

Le temple de Kashima ${ }^{5}$ (Kashima jingū 鹿島神宮) était considéré comme une sorte de passage, un point de transit entre le monde des hommes et celui des dieux. Une divinité guerrière du même nom, Kashima daimyōjin 鹿島大明神, y est célébrée, ainsi qu’un rocher monolithique censé, selon les croyances, maintenir le monde en place. Dans l'imaginaire du Japon d'alors, ce kanameishi 要石 ${ }^{6}$ servait de rempart, de défense contre les forces malveillantes de la nature. Les séismes ayant été très tôt considérés comme négatifs et rattachés dans l'imaginaire populaire aux mouvements d'un poisson-chat géant, il apparaît logique que par le biais des croyances on ait trouvé un moyen de combattre ce fléau, une force tout aussi puissante mais placée dans l'imaginaire du côté des hommes et du bien : la divinité Kashima et sa pierre de fondation semblaient alors parfaitement s'intégrer dans l'équation. Plus tard, on vit apparaître des motifs similaires à l'original, mais qui divergeaient toutefois sur certains points. Le rocher était parfois remplacé par une épée (fig. 1) qui semblait avoir le même effet sur le monstre, mais dont l'objectif premier était sans doute de focaliser l'attention sur la divinité toute-puissante

4. Seigneur de Fukuyama et gouverneur de la province d'Ise, membre du conseil du shogun à partir de 1843 .

5. Situé à Kashima, ville du département d'Ibaraki, à l'est de Tokyo.

6. Pierre de fondation, pierre rivet. 
et victorieuse acclamée par la population contrôlant le poisson avec une grande facilité . Ce postulat est d'ailleurs renforcé par quatre poissons-chats anthropomorphisés et agenouillés, soumis, qui semblent prier Kashima pour sa miséricorde. Au-delà de l'image de guerrier et de conquérant qui entoure le personnage de Kashima, il apparaît que cette symbolique du Dieu écrasant la Bête fait écho au récit mythique de la victoire de Susanoo no mikoto 素戔鳴尊8 sur le dragon Yamata no orochi 八岐大蛇 ${ }^{9}$. Ainsi, même si Amaterasu ōmikami 天照大神 ${ }^{10}$ était indéniablement restée ancrée dans l'imaginaire collectif comme une divinité puissante incarnée par l'empereur, à travers ces images, l'iconographie populaire réactualisait un des mythes les plus anciens de la culture japonaise ; certains symboles demeurant toujours aussi forts : la divinité salvatrice, le monstre effrayant et même l'épée ${ }^{11}$. À l'image de Susanoo dans les récits du Kojiki 古事記, Kashima était donc à son tour progressivement perçue dans l'iconographie populaire comme une divinité puissante proche du monde terrestre, en lien direct avec les humains, leur venant en aide.

Après le séisme d'Ansei, la peur de nouvelles secousses généra la production de talismans figurant des poissons-chats.

Ces imprimés, appelés plus tard namazu-e 鯰絵 ${ }^{2}$, représentaient la divinité Kashima reprenant le contrôle du monstre. L'un des plus typiques est

7. Le personnage semble en effet n'utiliser qu'une seule de ses mains.

8. Demi-frère d'Amaterasu, représentant les forces destructrices de la nature.

9. Dans la mythologie, dragon légendaire à huit têtes et huit queues qui désolait la province d'Izumo. Susanoo no mikoto l'aurait vaincu en le grisant avec du saké.

10. Littéralement : "Grande divinité illuminatrice du ciel », Kami principal des croyances shintô, symbolisant le soleil et la lumière.

11. On peut en effet voir une référence flagrante à l'épée légendaire murakumo no tsurugi 叢雲剣 trouvée dans la queue de Yamata no orochi par Susanoo no mikoto : «[... Lorsqu'il trancha la queue du milieu, la pointe de son épée se brisa. Trouvant cela étrange, il découpa la chair à l'aide du morceau cassé, regarda à l'intérieur et y découvrit une grande épée tranchante. Cette épée était la grande épée faucheuse d'herbe " (Chamberlain 1982 : tome I, section 18, "Le Serpent à huit fourches "). Cette épée deviendra ensuite un des trois symboles du pouvoir impérial puisqu'elle sera transmise à Ninigi no mikoto 瓊瓊杵尊 lors de sa descente sur terre : « [...] À leur arrivée, les divinités célestes firent don à l'auguste enfant de la parure de joyaux de huit pieds de long; ils lui donnèrent également la grande-épée-faucheuse-d'herbe [...] " (Chamberlain 1982 : tome I, section 33, "L'auguste descente des cieux du grand et auguste petit-fils »).

12. Littéralement : image de poisson-chat. 
appelé Jishin o-mamori 地震御守 ${ }^{13}$. Dans la figure 1, on remarque dans le coin supérieur gauche le nom Kashima dans un style spécifique associé à la symbolique taoïste ${ }^{14}$. Juste au-dessus est représentée la constellation de la Grande Ourse connue pour être un symbole de chance (Ōmori 1991 : 164-171).
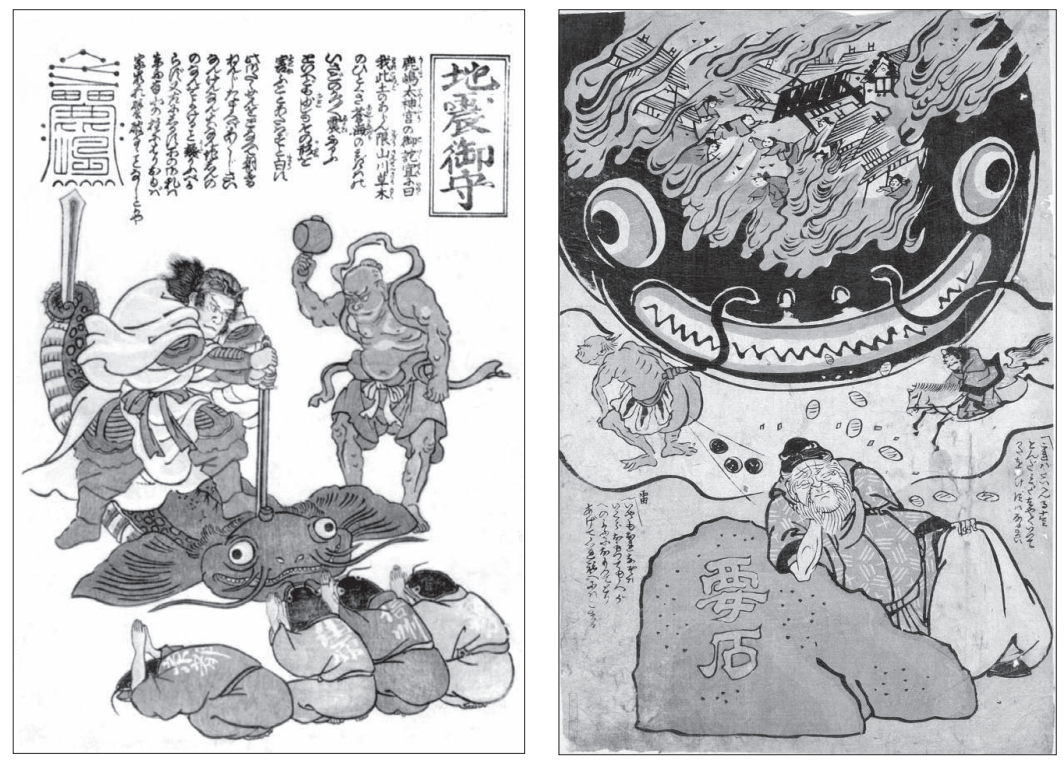

Fig. 1 (à gauche) «Talisman contre le tremblement de terre » (Jishin o-mamori 地震 御守). Collection du Musée d'histoire et de folklore du département de Saitama.

Fig. 2 (à droite) «Le poisson-chat et la pierre de fondation » (Namazu to kanameishi 鯰と要石) (1855). Collection de la Tokyo Metropolitan Library.

13. Littéralement : talisman protégeant des tremblements de terre.

14. Voir Miyata \& Takada (1995 : 110, 262, réf. 37). Collection du Musée d'histoire et de folklore du département de Saitama (Saitama kenritsu rekishi to minzoku no hakubutsukan 埼玉県立歴史と民俗の博物館). 
Face à ces images héroïques très répandues alors, il existait pourtant certains imprimés montrant le dégoût éprouvé à l'égard des divinités responsables du déséquilibre des forces cosmiques. Dans la figure 2, le feu fait rage et la terre tremble autour d'un sinistre poisson-chat géant ${ }^{15}$. Appuyé sur la pierre de fondation, la divinité Ebisu 恵比寿 ${ }^{16}$ semble fatiguée et somnole ${ }^{17}$. Parti hors de la ville, Kashima en cavalier à droite, rentre à la hâte. Sur la tête du namazu, Edo en proie aux flammes est la preuve que les divinités incompétentes ont permis à la catastrophe de se produire.

Ce dégoût provoqué par l'inaptitude de Kashima se manifestait parfois par le fait d'effacer la divinité en le remplaçant par Amaterasu. Avant le séisme d'Ansei, tout semblait indiquer que la population d'Edo avait peu d'intérêt pour la divinité tutélaire de la famille impériale, beaucoup plus connue à Kyoto. Cependant, dans certaines namazu-e, nous voyons apparaître les premiers indices d'une perception par les gens d'Edo d'Amaterasu comme une divinité salvatrice. La figure 3 en est un très bon exemple ${ }^{18}$. Le cheval blanc du temple d'Ise, un nusa 幣 $^{19}$ en guise de cavalier, chasse de ses sabots plusieurs poissons-chats; de fines lignes courbes entourent le cheval. Ce sont ses poils, censés représenter selon la croyance populaire la protection ultime contre le mal (Noguchi 2004 : 194). Kashima, en retrait à gauche dans son armure de samurai, tente sans succès de maîtriser l'animal.

15. Voir Miyata \& Takada (1995 : 266, réf. 44). Collection de la Tokyo Metropolitan Library (Tōkyō toritsu chūō toshokan 東京都立中央図書館) (réf. 東0277-C40) : <http:// www.library.metro.tokyo.jp/digital_library/collection/the28/no6/tabid/1690/Default. aspx> (dernière consultation : 18/04/2012).

16. Divinité populaire, considérée comme le Dieu du bonheur et de la prospérité, très vénérée par les pêcheurs et les commerçants.

17. Ebisu tient ici le rôle de rusugami 留守神, une divinité secondaire censée remplacer la divinité principale pendant son absence. Ce besoin aurait pour origine la croyance que toutes les divinités majeures devaient, chaque dixième mois, effectuer un voyage en Izumo. Cette période de l’année était appelée kannazuki 神無月 ou kaminazuki 神な月: "Le mois sans Dieu ». Pour plus de détails, voir Ouwehand (1964 : 16, 82-85).

18. Voir Miyata \& Takada (1995: 257-258, réf. 30). Collection Ishimoto, Bibliothèque de l'université de Tokyo (réf. 81-02-34) (Tōkyō daigaku fuzoku toshokan 東京大学附属 図書館, Jishin kasai hanga harimazechō Ishimoto korekushon I 地震火災版画張交帖石 本コレクションI) : <http://gazo.dl.itc.u-tokyo.ac.jp/ishimoto/2/02-034/00001.jpg> (dernière consultation : 01/04/2012).

19. Egalement appelé gohei 御幣 ou shimenawa 注連縄, symbole indiquant la nature sacrée du lieu et la présence d'un kami. 


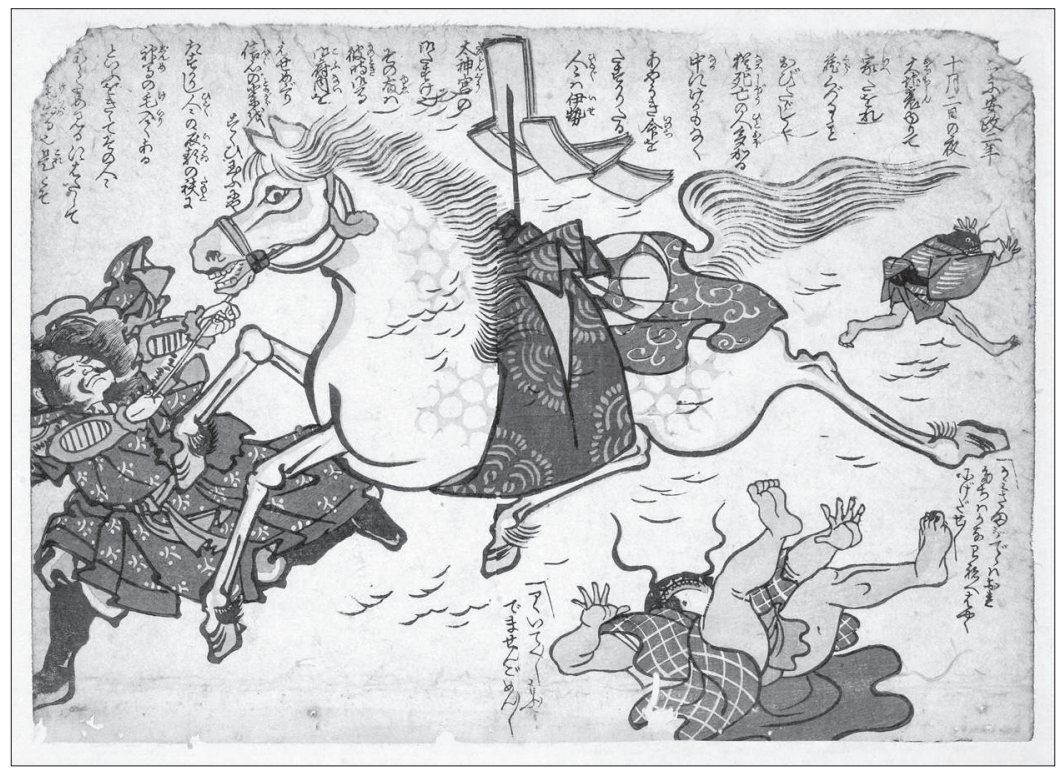

Fig. 3 " Le cheval du temple d'Ise dispersant les poissons-chats » (Namazu o kechirasu Ise-jingū jinme 鯰を踣散らす伊勢神宮神馬). Collection Ishimoto, Bibliothèque de l'université de Tokyo.

D'autres namazu-e montrent Kashima dans un rôle secondaire aux côtés d'Amaterasu, ou dans une relation antagoniste avec cette divinité. Dans la figure 4 par exemple ${ }^{20}$, Kashima, Amaterasu et Hachiman 八幡 ${ }^{21}$ chevauchent à travers le ciel, au-dessus d'Edo dévastée.

Amaterasu, précédée par Kashima, ordonne aux namazu de quitter les lieux sur l'instant, dispensant du crin ça et là. Ce dernier tient la pierre de fondation en l'air, mais celle-ci n'est d'aucune utilité dans le ciel. Dans cette

20. Voir Miyata \& Takada (1995 : 257, réf. 29). Collection de la bibliothèque nationale de la Diète (Kokuritsu kokkai toshokan 国立国会図書館) (réf. 7-8-2-6 00-0044) : <http://dl.ndl.go.jp/info:ndljp/pid/1302104> (dernière consultation : 01/04/2012).

21. Divinité populaire protectrice des guerriers et kami tutélaire de la famille Minamoto. Originaire de l'île de Kyūshū où son culte est attesté depuis le viII ${ }^{\mathrm{e}}$ siècle, elle fut, à partir de l'époque de Heian, identifiée à l'esprit de l'empereur semi-légendaire Ōjin. 


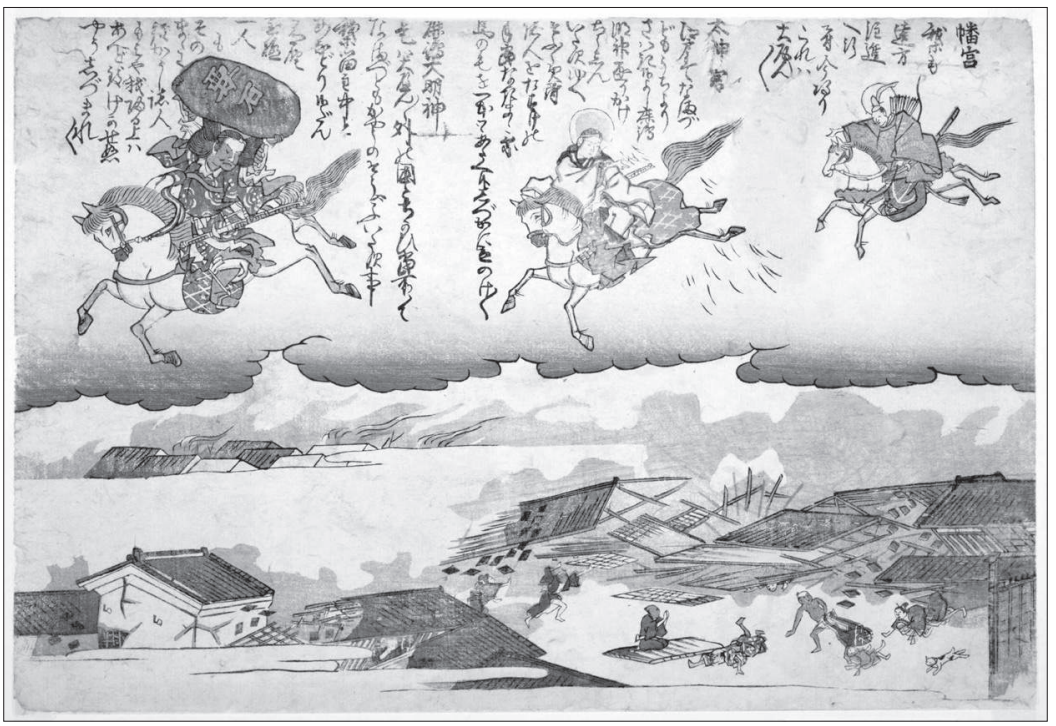

Fig. 4 " Hachiman, Amaterasu et Kashima galopant dans le ciel » (Amakakeru Hachimangū, Daijingū, Kashima daimyōjin 天駆ける八幡宮、太神宮、鹿島大明神). Collection de la bibliothèque nationale de la Diète.

image, Amaterasu et Kashima ont à peu près la même taille et sont positionnés à la même hauteur dans le ciel.

Dans la figure 5, Amaterasu, baptisée pour l'occasion « Divinité fondatrice de l'empire du grand Japon ", domine le personnage de Kashima, plus petit, qui l'assiste dans la distribution de crin de cheval sacré à l'instar de six autres divinités locales ${ }^{22}$. Ici, Kashima a été clairement rétrogradé. Le texte de l'image désigne le Japon comme le " pays des dieux " (shinkoku 神 国) dont les habitants ont la chance qu'Amaterasu et l'empereur s'inquiètent de leur bien-être (Miyata \& Takada 1995 : 258-260).

22. Voir Miyata \& Takada (1995 : 258-260, réf. 32). Institut historiographique de l'université de Tokyo (Tōkyō daigaku shiryō hensanjo 東京大学史料編纂所) (réf. 0380$18)$. 


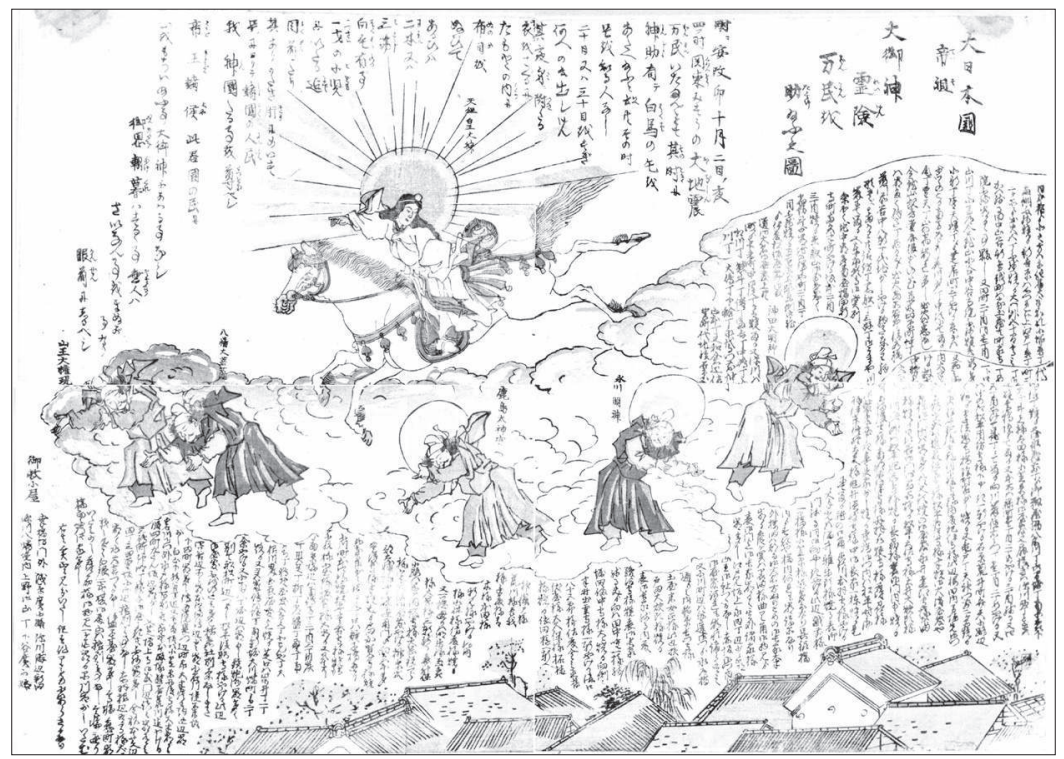

Fig. 5 « La puissance miraculeuse de la divinité fondatrice de l'empire du grand Japon secourant le peuple » (Dai Nihon koku teiso Ōmikami reigen banmin o tasuke tamau no zu 大日本国帝祖大御神霊験万民を助給ふ之図 ) Institut historiographique de l'université de Tokyo.

D'un côté, les habitants d'Edo semblent donc s'en remettre à Kashima pour reprendre le contrôle sur le poisson géant et rétablir l'équilibre des forces cosmiques ; de l'autre, même dans les namazu-e les moins explicites, la critique envers Kashima est évidente et il apparaît clairement que dans l'imaginaire collectif cette divinité est en grande partie responsable des destructions, car elle a échoué dans l'accomplissement de sa mission. En effet, le tremblement de terre d'Ansei eut lieu le $10^{\mathrm{e}}$ mois de l'année selon le calendrier traditionnel (novembre selon le calendrier occidental). Il est nécessaire de rappeler que selon la croyance populaire ce mois était aussi appelé kannazuki 神無月23 ou " mois sans divinité ». Kashima, à l'instar des autres dieux du panthéon shintô, devait partir à Izumo où tous

23. Pour plus de détails, voir Jean-Michel Butel et Pascal Griolet (1999). 
se réunissent. La divinité absente au moment de la catastrophe était alors dans l'incapacité d'agir. C'est pourquoi, face à ce dieu faillible, imparfait, aux caractéristiques définitivement humaines, il semble que la réponse iconographique ait été de remplacer Kashima et de se tourner ainsi vers la toute-puissance salvatrice d'Amaterasu ${ }^{24}$. Dans la réalité, grâce à l'influence grandissante des namazu-e, ce face à face entre héros guerrier et divinité fondatrice semble s'être rapidement calqué dans l'inconscient collectif sur les conflits qui faisaient rage à l'époque entre maison impériale et shogunat. Ainsi, même si ce n'est que très indirectement, dans l'imaginaire de la population, les namazu-e nées de la catastrophe d'Ansei préfigurent l'affaiblissement, puis le renversement du régime shogunal des Tokugawa et la restauration impériale de 1867 .

Au lendemain du 11 mars 2011, dans la souffrance et le dénuement le plus total, les régions du nord-est du Japon endeuillées ont commencé un long processus de reconstruction. Face à la catastrophe, responsables politiques et grands patrons ont très rapidement été les cibles des plus vives critiques de la part de la population ; certains, pour leurs propos déplacés ou leur mauvaise gestion de la situation, furent directement ou indirectement poussés à la démission, comme le Premier ministre Kan Naoto 菅直人, le président de Tepco Shimizu Masataka 清水正孝 ou plus tardivement le ministre de l'Économie Hachiro Yoshio 鉢呂吉雄 ${ }^{25}$. Au milieu du tumulte social et médiatique, la voix de l'empereur s'est fait entendre, d'abord à la télévision, puis au chevet des victimes (Hishitsuka 2011). Alors que les Japonais l'avaient pour la plupart, depuis de nombreuses années, relégué à un simple symbole, le souverain semble avoir profondément touché l'opinion publique du Japon par ses déclarations. Peut-on penser pour autant que l'empereur est amené à jouer un rôle politique qu'il n'avait pas jusqu’à

24. Ceci rappelle une nouvelle fois les récits mythiques dans lesquels Amaterasu est clairement favorisée au profit de Susanoo à de nombreuses reprises : "Il le remit à la grande-et-auguste-divinité-ciel-flamboyant et lui dit : "que votre auguste personne gouverne la plaine-des-hauts-cieux” [...]»(Chamberlain 1982 :tome I, section 11). "Alors que les deux autres divinités assumaient les responsabilités données par leur père, l'auguste-mâle-rapide-impétueux refusa de gouverner le royaume dont on lui avait donné la charge [...] il fut alors banni. " (ibid. : tome I, section 12).

25. Voir, entre autres, Mougey (2011), AFP (2011). 
présent? Ou bien d'autres forces politiques nouvelles sont-elles à l'œuvre? $\grave{A}$ travers cette situation post-traumatique que vit actuellement le pays, assisterions-nous comme en un écho de la catastrophe de 1855 aux prémisses d'un bouleversement politique?

27 juin 2011

\section{Bibliographie}

AFP, 2011

"Le ministre japonais de l'Économie démissionne après Fukushima ", dépêche du 10 septembre.

\section{BUTEL Jean-Michel}

\section{\& GRIOLET Pascal, 1999}

"Histoires de poissons-chats:

les images du grand séisme de 1855

à Edo », Ebisu, 21 (numéro spécial « Le Japon des séismes ») : 17-32.

CHAMBERLAIN Basil H. (trad.), [1883] 1982

The Kojiki. Records of Ancient Matters, Boston, Tokyo, Tuttle Publishing.

\section{FRÉDÉRIC Louis, 1996}

Le Japon. Dictionnaire et civilisation, Paris, Robert Laffont, Collection Bouquins.

HISHITSUKA Hiroshi 菱塚博, 2011 «Tennō/kōgō ryōheika, Fukushima iri Tōhoku no hisaichi rekihō sankasho me »天皇·皇后両陛下、福島入り 東北
の被災地歴訪3力所目 (L'empereur et l'impératrice à Fukushima. Troisième visite d'un site sinistré dans le Tōhoku), Asahi shinbun 朝日新聞, 11 mai.

MIYATA Noboru 宮田登

\& TAKADA Mamoru 高田衛 (dirs.), 1995 Namazu-e. Shinsai to Nihon bunka 鯰絵一一震災と日本文化 (Namazu-e. Les désastres sismiques et la culture japonaise), Tokyo, Ribun shuppan 里文出版.

MOUGEY Amélie, 2011

"Le Premier ministre paie Fukushima », Ouest-France, 5 août.

NOGUCHI Takehiko 野口武彦, 2004 Ansei Edo jishin 安政江戸地震 (Le séisme d'Edo de l'ère Ansei), Tokyo, Chikuma shobō 筑摩書房.

ŌMORI Takashi 大森隆, 1991

Furō fushi o mezasu sendō shukujutsu no sekai 不老不死を目指す先導祝術の世界 
(Le monde des techniques ésotériques destinées à atteindre l'immortalité), Tokyo, Dōkyō no hon 道教の本.

Kojiki 古事記 (Récit des choses anciennes), [712] 1997

in Shinpen Nihon koten bungaku zenshū 新編日本古典文学全集 (Collection complète de la littérature classique du Japon, nouvelle édition), t. 1, Tokyo, Shōgakukan 小学館.

Nihon shoki 日本書紀 (Chroniques du Japon), [720] 1998 in Shinpen Nihon koten bungaku zenshū 新編日本古典文学全集 (Collection complète de la littérature classique du Japon, nouvelle édition), t. 4, Tokyo, Shōgakukan 小学館.

\section{OUWEHAND Cornelis, 1964}

Namazu-e and their Themes: An Interpretative Approach to Some Aspects of Japanese Folk Religion, Leiden, E.J. Brill.

USAMI Tatsuo 守佐美龍夫, 2003 Nihon higai jishin sōran 日本被害地震 総覧 (Histoire générale des séismes destructeurs au Japon), Tokyo, Kōwa shuppan 恒和出版.

WAKAMIZU Suguru 若水俊, 2003 Namazu wa odoru : Edo no namazu-e omoshiro bunseki 鯰は踊る——江戸の 鯰絵面白分析 (Le poisson-chat danse: analyse de l'humour des namazu-e de l'époque d'Edo), Tokyo, Bungeisha 文芸社. 